\title{
CircRNA_101505 sensitizes hepatocellular carcinoma cells to cisplatin by sponging miR-103 and promotes oxidored-nitro domain-containing protein 1 expression
}

\author{
Yanwei Luo ${ }^{1}$, Yunfeng Fu', Rong Huang ${ }^{1}$, Meng Gao ${ }^{1}$, Fengxia Liư ${ }^{1}$ Rong Gui ${ }^{1}$ and Xinmin $\mathrm{Nie}^{1}$
}

\begin{abstract}
Hepatocellular carcinoma (HCC) is one of the most common malignant tumors and a leading cause of cancer-related deaths worldwide. Emerging studies have shown that circular RNAs (circRNAs) are differentially expressed in HCC and play an important role in HCC pathogenesis and metastasis. However, the mechanism of circRNA in the chemoresistance of HCC remains unclear. In this study, we aimed to investigate the role of circRNA in cisplatin resistance of HCC. We identified a novel circRNA circRNA_101505 that was decreased in cisplatin-resistant HCC tissues and cell lines, and associated with a poor survival outcome. Gain-of-function investigations showed that overexpression of circRNA_101505 suppressed cancer cell growth in vivo and in vitro, and enhanced cisplatin toxicity in HCC cells. Mechanistic studies found that circRNA_101505 could sensitize HCC cells to cisplatin by sponging miR103, and thereby promoting oxidored-nitro domain-containing protein 1 (NOR1) expression. In conclusion, the significant inhibitory effects indicate circRNA_101505 to be a potential therapeutic target for HCC treatment. Our findings provide significant evidence to further elucidate the therapeutic use of circRNA in HCC.
\end{abstract}

\section{Introduction}

Hepatocellular carcinoma (HCC) is one of the most common malignant tumors and a leading cause of cancerrelated deaths worldwide ${ }^{1}$. Owing to the high frequency of tumor metastasis, recurrence, and drug resistance, the survival rate of patients with HCC is low. Additionally, the effective therapies for advanced HCC patients are limited $^{2}$. Thus, identifying novel targets for HCC therapy is urgently required. Emerging studies have found that circular RNAs (circRNAs) are differentially expressed in HCC and play an important role in HCC pathogenesis and metastasis ${ }^{3}$.

Correspondence: Fengxia Liu (592111443@qq.com) or Rong Gui (aguirong@163.com)

'Department of Blood Transfusion, the Third Xiangya Hospital of Central South University, Tongzipo Road 138, 410013 Changsha, China

Edited by I. D'Agnano
CircRNAs are a tissue-specific class of noncoding RNAs molecules. They are characterized by a covalently closed continuous loop without poly(A) tail ${ }^{4}$. Recently, thousands of endogenous circRNAs have been discovered. CircRNAs mediate gene expression by sponging microRNAs or interacting with other molecules and then inhibit their function ${ }^{5}$. For example, circRNA, circZNF652 promotes migratory and invasive capabilities of HCC cells by sponging miR-203 and miR-502-5p and enhancing epithelial-mesenchymal transition (EMT) ${ }^{6}$. CircRNA circSETD3 was significantly downregulated in HCC tissues and cell lines and associated with unfavorable prognosis of HCC patients ${ }^{7}$. Matboli et al showed that the combination of hsa_circ_00156, hsa_circ_00224 and hsa_circ_00520 acted as biomarkers with higher sensitivities and specificities than alpha-fetoprotein in $\mathrm{HCC}^{8}$. Multidrug resistance occurs frequently in $\mathrm{HCC}$ during the long-term chemotherapy, leading to cancer

\section{(c) The Author(s) 2019}

(c) Open Access This article is licensed under a Creative Commons Attribution 4.0 International License, which permits use, sharing, adaptation, distribution and reproduction cc) in any medium or format, as long as you give appropriate credit to the original author(s) and the source, provide a link to the Creative Commons license, and indicate if changes were made. The images or other third party material in this article are included in the article's Creative Commons license, unless indicated otherwise in a credit line to the material. If material is not included in the article's Creative Commons license and your intended use is not permitted by statutory regulation or exceeds the permitted use, you will need to obtain permission directly from the copyright holder. To view a copy of this license, visit http://creativecommons.org/licenses/by/4.0/. 
relapse. An increasing number of studies have highlighted the key roles of ncRNAs, including miRNAs, long ncRNAs (lncRNAs) in chemoresistance of $\mathrm{HCC}^{9}$. However, the mechanism of circRNA in chemoresistance of HCC remains unclear.

In this study, we aimed to investigate the role of circRNA in cisplatin resistance of HCC. We identified a novel circRNA circRNA_101505, that was decreased in cisplatin-resistant HCC tissues and cell lines. Gain-offunction investigations showed that circRNA_101505 overexpression suppressed cancer cell growth in vivo and in vitro. Subsequent studies displayed that circRNA_101505 could sensitize HCC cells to cisplatin by targeting the miR-103/oxidored-nitro domain-containing protein 1 (NOR1) signaling axis.

\section{Results}

\section{Downregulation of CircRNA_101505 is associated with} cisplatin resistance in $\mathrm{HCC}$

To investigate the role of circRNAs in cisplatin-resistant HCC, we performed circRNAs array to identify the differentially expressed circRNAs. Several circRNAs were differentially expressed in cisplatin-resistant and -sensitive HCC (Fig. 1a). qPCR results further confirmed that circRNA_101505 was significantly decreased in HCC tissues compared with adjacent tissues, and its expression was lower in cisplatin-resistant HCC tissues than in the cisplatin-sensitive tissues (Fig. 1b). We also found that circRNA_101505 was significantly decreased in cisplatinresistant HCC cell lines (Hep3B-R and Huh7-R) compared with the parental HCC cell lines (Fig. 1c). In addition, further survival analyses revealed that the HCC patients with low circRNA_101505 level had shorter overall survival than the patients had high circRNA_101505 level $(p=0.0005$, Fig. 1d). These results suggest that circRNA_101505 is closely associated with cisplatin resistance in $\mathrm{HCC}$.

\section{Overexpression of circRNA_101505 sensitizes HCC cells to cisplatin}

To investigate the role of circRNA_101505 in cisplatin resistance in HCC, we overexpressed circRNA_101505 in Hep3B-R and Huh7-R cells (Fig. 2a). We found that overexpression of circRNA_101505 significantly inhibited cells proliferation (Fig. 2b) and induced apoptosis (Fig. 2c) in Hep3B-R and Huh7-R cells compared with negative control. In addition, circRNA_101505 upregulation sensitizes Hep3B-R and Huh7-R cells to cisplatin (Fig. 2d, e). Furthermore, the tumor suppressive effects of circRNA_101505 upregulation were also confirmed in vivo. Our results showed that the tumor volumes in nude mice injected with Hep3B-R cells expressing circRNA_101505 were smaller than in the control nude mice (Fig. 2f), and the proliferative marker ki67 expression was decreased in tumor tissues that expressing circRNA_101505 compared with control (Fig. 2g).

\section{CircRNA_101505 sponges miR-103 in HCC}

We tested whether circRNA_101505 binds to miRNAs in HCC cells. The bioinformatics tool predicted two binding sites on circRNA_101505 for miR-103 (Fig. 3a). Further luciferase assay confirmed that circRNA_101505 targeted to miR-103 (Fig. 3b), suggesting that circRNA_101505 may function as a sponge to miR-103. In addition, RIP assay revealed that miR-103 directly interacted with circRNA_101505 (Fig. 3c). To investigate the downstream mechanism by which circRNA_101505 exerted its functions in HCC cells, the mimic of miR-103 was co-transfected with circRNA_101505. The results showed that miR-103 mimic could significantly reversed circRNA_101505-mediated induction of apoptosis (Fig. 3d, e) and promotion of cisplatin toxicity in Hep3B-R and Huh7-R cells (Fig. 3f).

\section{MiR-103 confers cisplatin resistance in HCC cells by targeting NOR1}

We further investigated the role and downstream mechanism that miR-103 affects cisplatin resistance in HCC. NOR1 was a predictive target of miR-103 (Fig. 4a). MiR-103 wild-type and mutant sequences were constructed into luciferase reporter gene and co-transfected with NOR1, and luciferase assay confirmed that miR-103 targeted to NOR1 (Fig. 4a, b), and negatively regulated NOR1 expression (Fig. 4c). We expressed NOR1 in Hep3B-R and Huh7-R cells (Fig. 4d), and found that NOR1 could significantly reversed miR-103-mediated inhibition of apoptosis and suppression of cisplatin toxicity in Hep3B-R and Huh7-R cells (Fig. 4e, f). In addition, we also found that the levels of miR-103 were significantly increased and NOR1 was decreased in HCC tissues compared with adjacent tissues (Fig. 5a), and miR-103 levels were higher but NOR1 levels were lower in cisplatin-resistant HCC tissues than in the cisplatinsensitive tissues (Fig. 5a). Pearson analysis showed that circRNA_101505 were negatively correlated with miR-103 (Fig. 5b) and positively correlated with NOR1 (Fig. 5c), and miR-103 was negatively correlated with NOR1 (Fig. 5d). These results demonstrate that circRNA_101505 sensitizes HCC cells to cisplatin through miR-103/NOR1 axis.

\section{Discussion}

In this study, we found that circRNA_101505 downregulation is associated with a poor survival outcome and cisplatin resistance in HCC. Functional investigations revealed that circRNA_101505 overexpression inhibited HCC cell growth and sensitized HCC cells to cisplatin. Further experiments demonstrated that circRNA_101505 


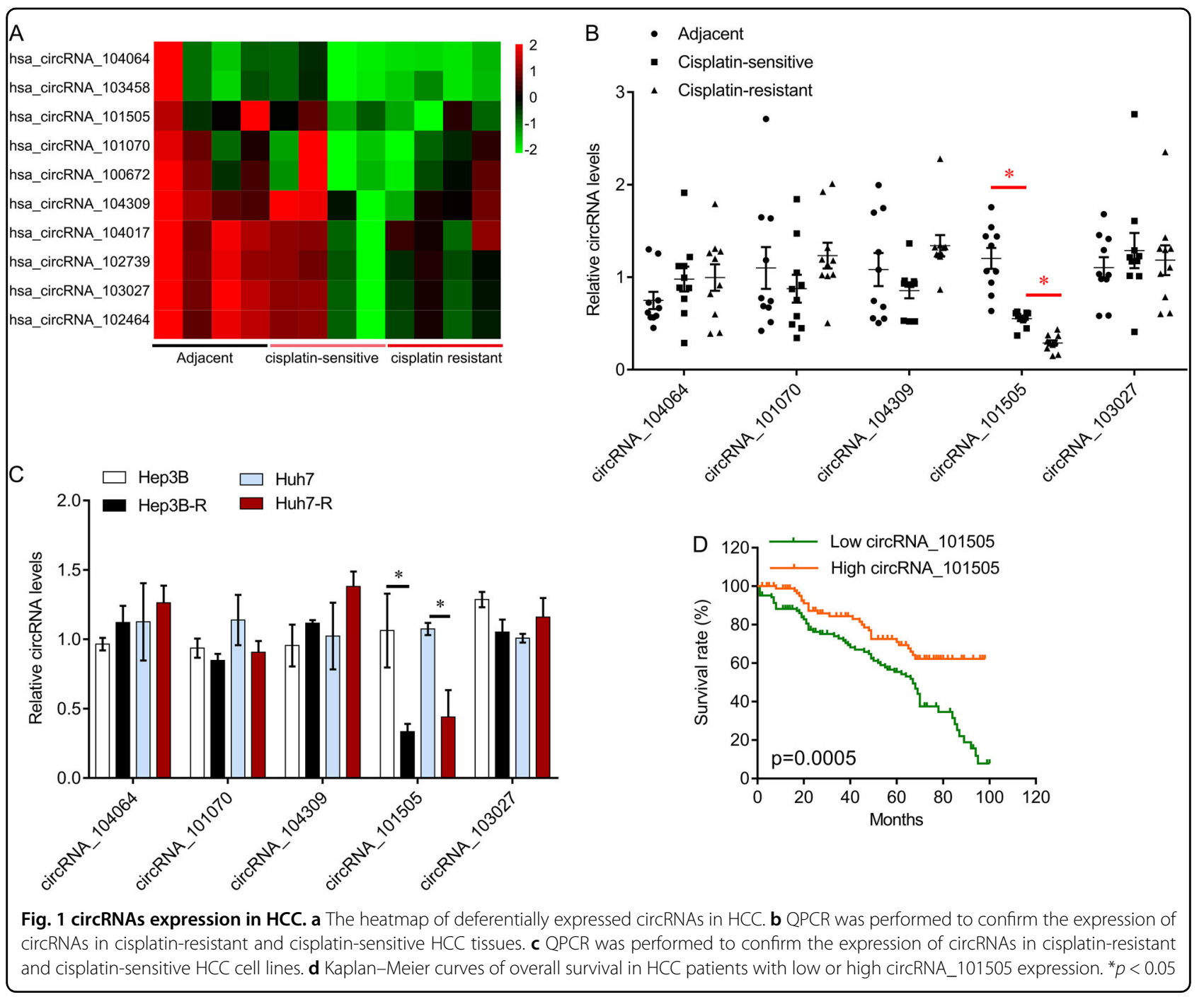

exhibited tumor suppressive effects by sponging miR-103, leading to NOR1 upregulation.

Cisplatin is a small-molecule platinum compound. Cisplatin-based chemotherapeutic regimens are one of the most widely used regimens for the treatment of various solid cancers, including liver cancer, cervical cancer and several others. The anticancer activity of cisplatin involves multiple mechanisms ${ }^{10}$. However, drug resistance of cisplatin limits its application and effectiveness. Cisplatin resistance may result from reduced drug accumulation inside cancer cells and drug inactivation by fast repairing of DNA lesions ${ }^{11}$. Several efforts have been made to minimize cisplatin resistance ${ }^{12}$. Recently, new evidence shows that circRNAs are associated with cisplatin resistance ${ }^{13}$. For example, upregulation of circPVT1 was observed in osteosarcoma tissues, serums and cisplatin-resistant cell lines, and was correlated with poor prognosis of patients with osteosarcoma. circPVT1 knockdown sensitizes osteosarcoma cells to cisplatin by decreasing ABCB1 expression ${ }^{14}$. The expression of circAKT3 was upregulated in cisplatin-resistant gastric cancer tissues and cells compared with cisplatin-sensitive samples, and was associated with aggressive characteristics and poor survival outcomes. CircAKT3 promotes DNA damage repair and inhibits apoptosis through PIK3R1 activation by sponging miR-198 ${ }^{15}$. Chi et al. have reported that hsa_circ_0000285 expression is lower in patients with cisplatin-resistant bladder cancer, and acts as an independent prognostic factor for bladder cancer patient outcome ${ }^{16}$. In this study, we demonstrated that circRNA_101505 was downregulated in HCC tissues and associated with poor survival outcomes and cisplatin resistance in HCC. Gain-of-function experiments revealed that circRNA_101505 overexpression inhibited HCC cell proliferation, induced apoptosis and sensitized HCC cells to cisplatin in vitro and in vivo.

Further investigations showed that circRNA_101505 interacted with miR-103, and miR-103 mimics reversed 


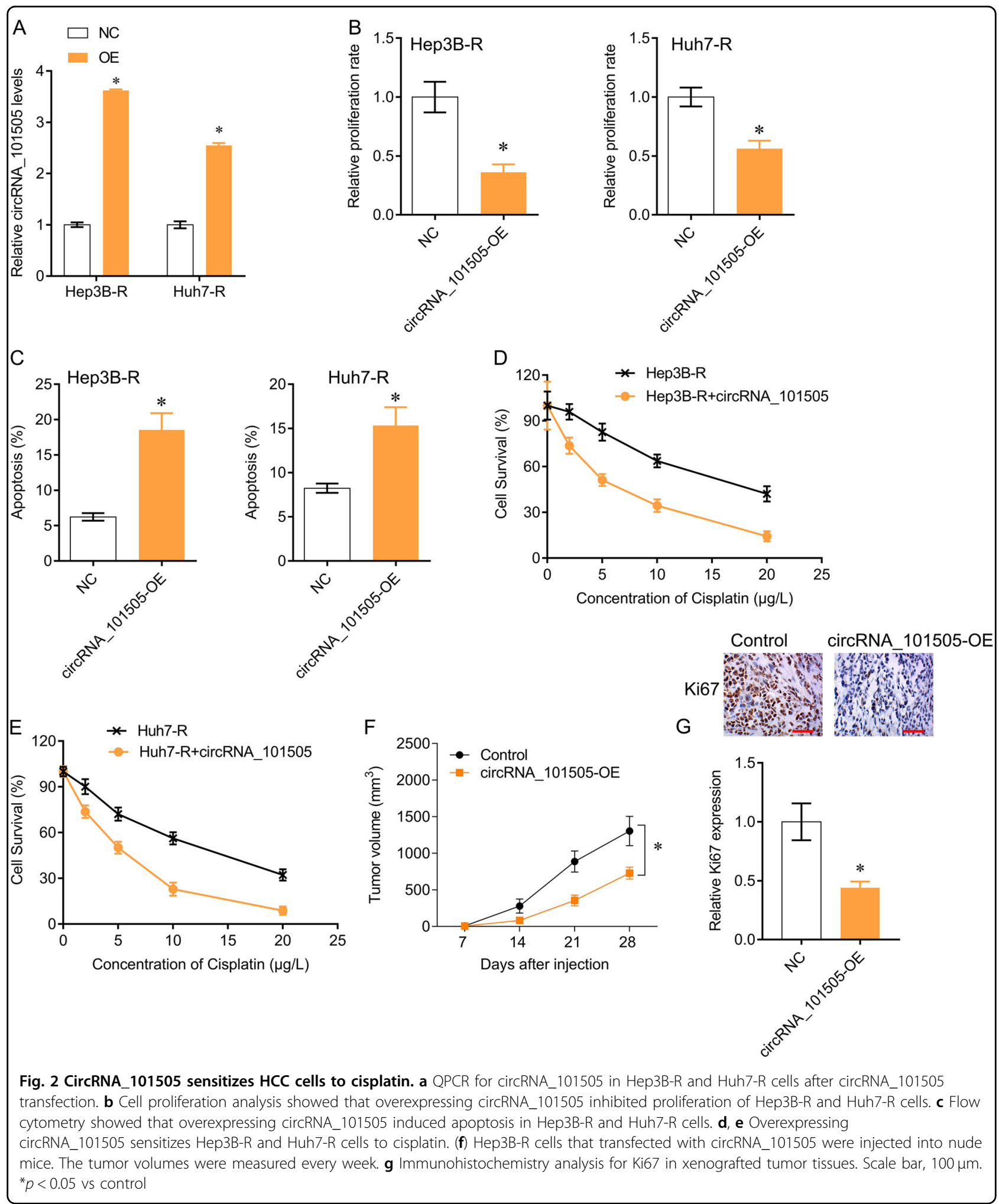

circRNA_101505-mediated tumor suppressive effects. Noncoding RNAs, including miRNAs, lncRNAs and circRNAs, play an important role in the evolution and progression of drug resistance in cancers. The mechanisms are associated with drug transporter-related proteins, cell apoptosis-related proteins, DNA damage repair, and tumor microenvironment ${ }^{17}$. MiR-103 expression is upregulated in Adriamycin-resistant acute myeloid 


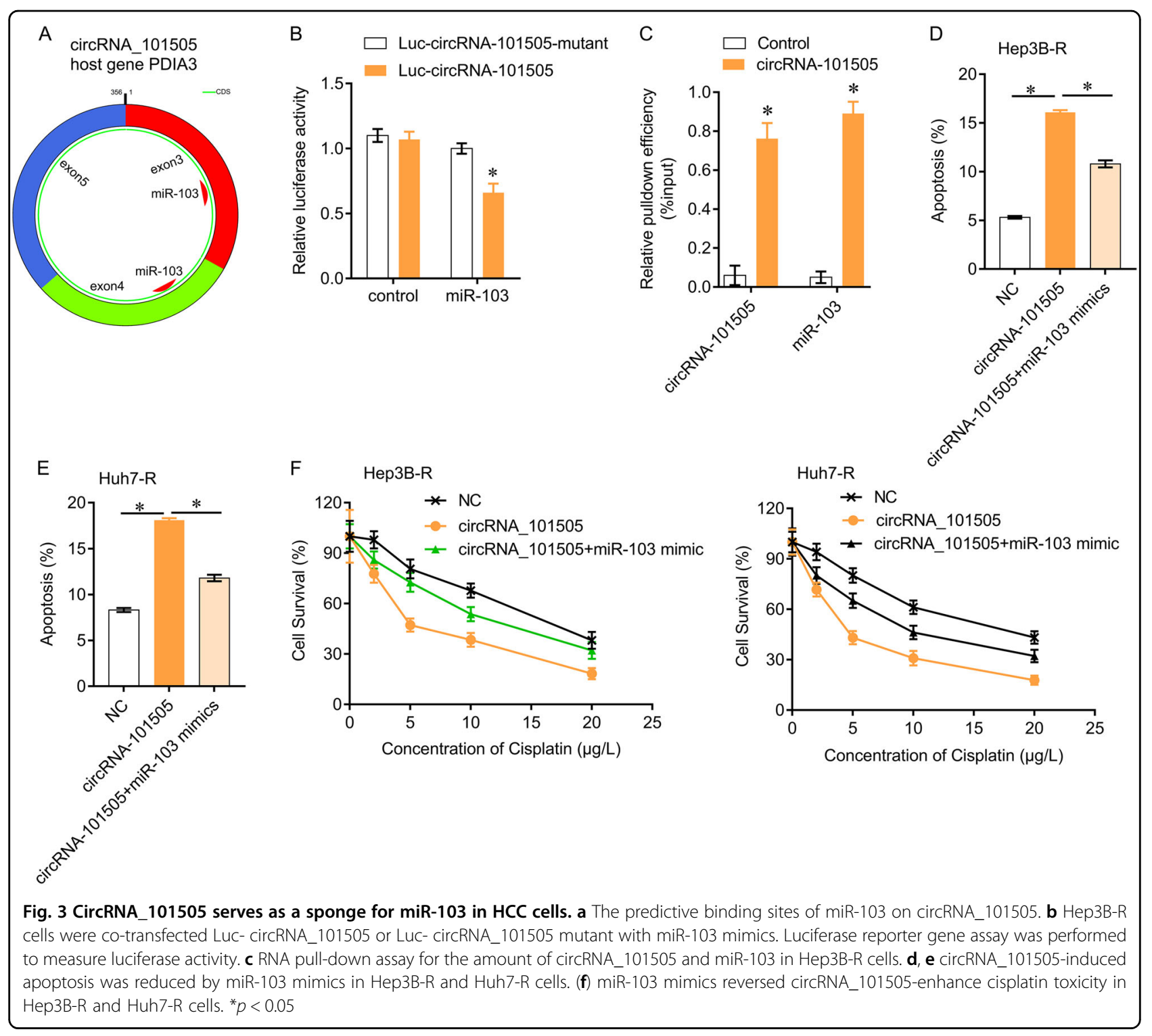

leukemia cells and confers K562 cells' drug resistance via regulation of COP1 through PI3K/AKT signal pathway ${ }^{18}$. MiR-103 is controlled only by the MET oncogene and associated with gefitinib-induced apoptosis and epithelialmesenchymal transition of non-small cell lung cancers in vitro and in vivo ${ }^{19}$. Furthermore, a recent study also demonstrates that circular RNA circARF3 acts as an endogenous miR-103 sponge to alleviate adipose inflammation by promoting mitophagy ${ }^{20}$.

In this study, we identified circRNA_101505 as a new interactive molecule of miR-103, also confirmed that NOR1 was a new downstream target of miR-103. NOR1 was cloned in our previous study and was involved in the development and progression of nasopharyngeal carcinoma ${ }^{21,22}$. Moreover, it inhibited proliferation and induced apoptosis via the MAPK activation in prostate cancer cells ${ }^{23}$. In hepatocellular carcinoma, we previously demonstrated that NOR1 increases the expression of growth factor receptor-bound protein 2 (Grb2) mRNA in the human hepatocellular carcinoma cells, and NOR1 enhanced monofunctional alkylating agent 5-(aziridin-1yl)-2,4-dinitrobenzamide (CB1954)-induced cell killing in HCC cells, but this effect was reversed by stable transfection of Grb2 small hairpin RNA ${ }^{24}$. These results indicate that circRNA_101505 functions as a tumor suppressor by sponging miR-103, leading to NOR1 upregulation, and finally inhibits cell growth and sensitizes HCC cells to cisplatin.

In conclusion, our study revealed that circRNA_101505 is frequently inactivated in cisplatin-resistant HCC tissues and cell lines and associated with a poor survival outcome. CircRNA_101505 may sponge miR-103 and thereby 


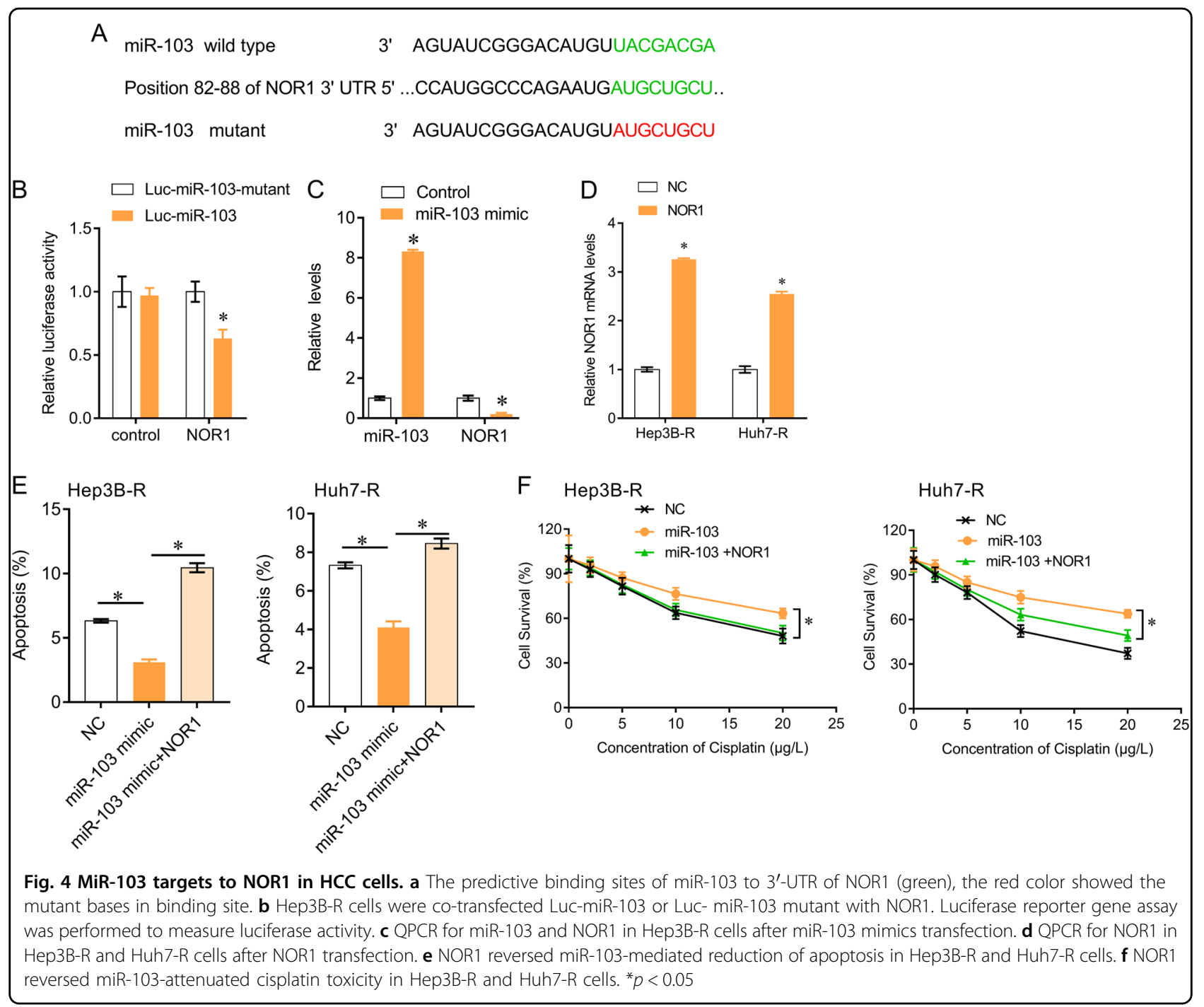

promote NOR1 expression. Subsequently, this may suppress cell growth and sensitize HCC cells to cisplatin in vitro and in vivo. The significant inhibitory effects indicate circRNA_101505 as a potential therapeutic target for HCC treatment. Therefore, our findings provide significant evidence to further elucidate the therapeutic use of circRNA in HCC.

\section{Material and methods}

\section{Human tissues collection}

This project was approved by the Ethic Committee of The Third Xiangya Hospital of Central South University. Written informed consent was obtained from each patient. Hepatocellular carcinoma tissues $(N=120)$ and the adjacent tissues $(N=78)$ were collected from The Third Xiangya Hospital of Central South University between May 2008 and Nov 2018. Each sample was immediately flash-frozen in liquid nitrogen until RNA extraction.

\section{CircRNA microarray analysis}

Total RNA was extracted from patients with cisplatinresistant or cisplatin-sensitive HCC using the RNeasy Mini Kit (Qiagen, GmBH, Hilden, Germany) according to the manufacturer's instructions. The adjacent tissues were used as control. Patients with cisplatin-resistant HCC were defined as those with persistent disease more than two months, and those with recurrent disease more than 2 months after completion of chemotherapy containing cisplatin. Patients with cisplatin-sensitive HCC were defined as those without local residual lesions or recurrence at 2 months after completion of chemotherapy containing cisplatin. Purified total RNA was quantified using the NanoDrop 2000 spectrophotometer. The total RNA was sent to Aksomics Co. Ltd. (Shanghai, China) to analyze circRNA expression profiles. Differentially expressed circRNAs were identified as fold change $>2$ and adjusted $\mathrm{p}<0.05$. 

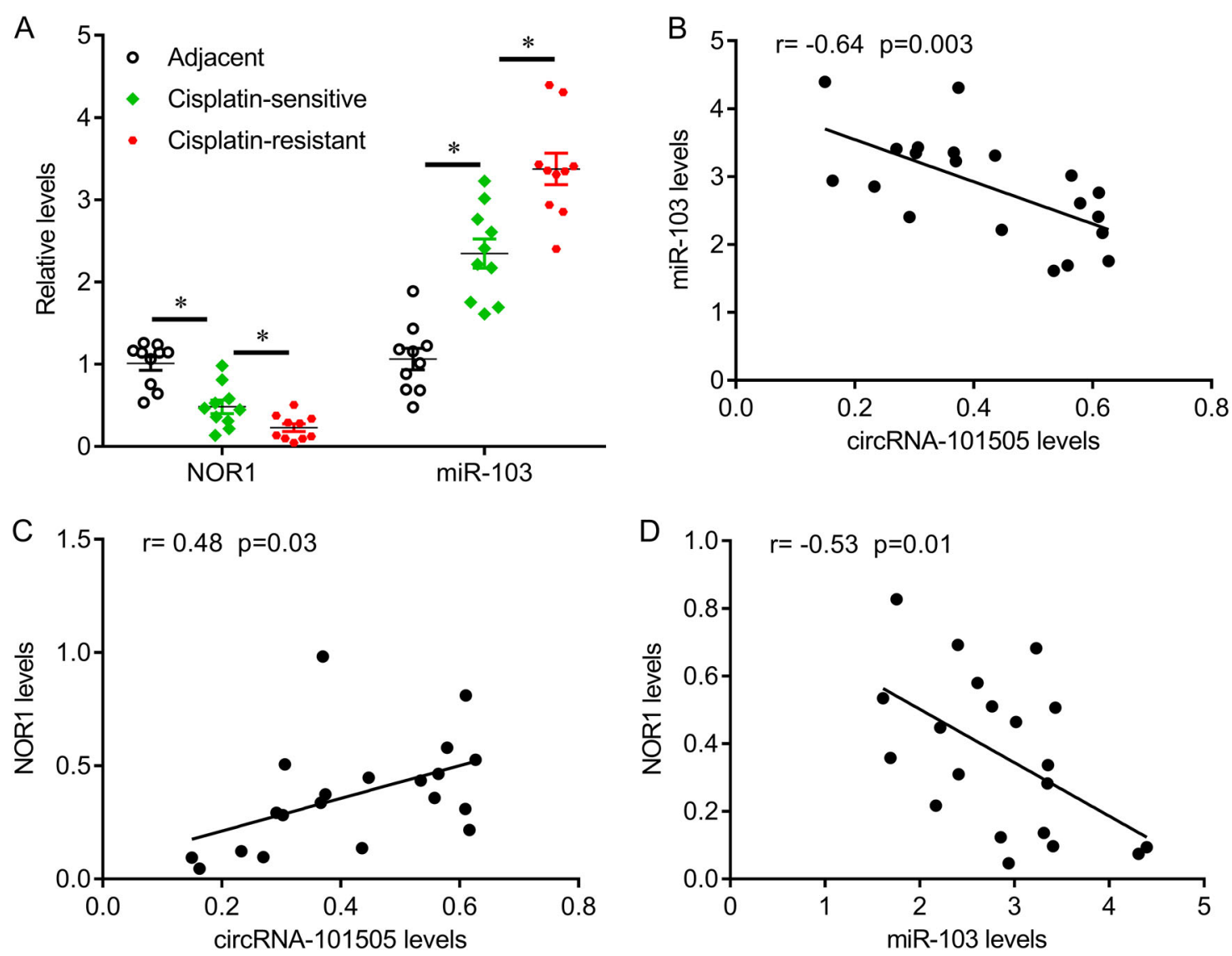

Fig. 5 The relationship of circRNA_101505, miR-103 and NOR1 in patients with HCC. a Scatter plots illustrating qRT-PCR analysis of expression fold change for miR-103 and NOR1 in cisplatin-resistant and cisplatin-sensitive HCC tissues. b circRNA_101505 expression was negatively correlated with miR-103. c circRNA_101505 expression was positively correlated with NOR1. d miR-103 expression was negatively correlated with NOR1. ${ }^{*} p<0.05$ vs control

\section{Cell culture}

The cell lines used in the present study included Hep3B and Huh7 cells were cultured in Roswell Park Memorial Institute (RPMI) 1640 medium (Gibco) supplemented with $10 \%$ fetal bovine serum and maintained in a humidified atmosphere with $5 \% \mathrm{CO}_{2}$ at $37^{\circ} \mathrm{C}$.

The cisplatin-resistant Hep3B (Hep3B-R) and Huh7 (Huh7-R) cells was established in our lab. The Hep3B or Huh7 cells were treated with the initial concentration of cisplatin $(0.5 \mu \mathrm{g} / \mathrm{L})$. The medium was changed once every 3 days. Each dose was maintained for 2 weeks, and then was doubled.

To test the cell survival and proliferation rates, Hep3B$\mathrm{R}$ and Huh7-R cells were exposed to cisplatin at a series of concentrations $(0,2.5,5,10$ and $20 \mu \mathrm{g} / \mathrm{L})$ for $24 \mathrm{~h}$ or at $10 \mu \mathrm{g} / \mathrm{L}$ for $24 \mathrm{~h}$.

\section{Cell transfection}

circRNA_101505 overexpression plasmids (circRNA_101505-OE) and NOR1 expressed plasmids were constructed and purchased from (GenomediTech, Shanghai, China). miR-103 inhibitors and mimics were purchased from RiboBio (Guangzhou, China). Transfection was performed using Lipofectamine 3000 (LifeTechnologies, USA) according to the manufacturer's instructions.

\section{Cell proliferation analysis}

A BrdU Cell Proliferation Assay Kit (CST (Shanghai) Biological Reagents Company Limited, Shanghai, China) was used to measure the cell proliferation rate according to the manufacturer's instructions. The cells were seeded in a 96-well plate and incubated overnight. The cells then were transfected circRNA_101505, miR-103 or NOR1, with co-treatment of cisplatin and cells were incubated for $24 \mathrm{~h}$. Finally, $10 \mu \mathrm{M}$ BrdU was added to the plate and cells were incubated for $4 \mathrm{~h}$. The absorbance at $450 \mathrm{~nm}$ was read and recorded to calculate the relative proliferation rate. The experiment was done three times with triplicate samples. 


\section{Cell apoptosis analysis}

An Annexin V-FITC Apoptosis Detection Kit (CST (Shanghai) Biological Reagents Company Limited, Shanghai, China) was used for analysis of apoptosis according to the manufacturer's instructions. Briefly, cells with indicated treatment were resuspended in $236 \mu \mathrm{l}$ Annexin V-FITC binding buffer and then mixed well with $1 \mu \mathrm{l}$ Annexin V-FITC and $13 \mu \mathrm{l}$ Propidium Iodide for $30 \mathrm{~min}$ incubation. The cells were analyzed by using a BD FacScanto II flow cytometer (BD Biosciences, San Jose, CA). Unstained cells, and fluorescence minus one (FMO) controls were used for cytometry and gating set up.

\section{Quantitative PCR analysis}

Total RNA was extracted with Trizol reagent. The expression of circRNA_101505 and NOR1 was measured by TaqMan Fast Advanced Master Mix (Thermo Scientific, Shanghai, China) according to manufacturers' instructions. Expression of $\beta$-actin was used as an endogenous control. QPCR was performed at the condition: Hold $50^{\circ} \mathrm{C}$ for $2 \mathrm{~min}, 95.0^{\circ} \mathrm{C}$ for $20 \mathrm{~s}$, and 40 circles of $95.0^{\circ} \mathrm{C}$ for $1 \mathrm{~s}$ and $60^{\circ} \mathrm{C}$ for $20 \mathrm{~s}$ in 7900 HT Fast RealTime PCR Instrument. The primers were used as following: circRNA_101505: forward, 5'-CCGAGTTCCTAA AAGCAGCC-3'; reverse, 5'-CCATCAGCAGTCCTAG GTCC-3'. $\beta$-actin: forward, 5'-CACACTGTGCCCATCTATGAGG-3'; reverse, 5'-TCGAAGTCTAGGGCGACATAGC-3' ${ }^{\prime}$. miR-103 expression was measured by miRNA QPCR Master Mix (Agilent, Beijing, China) according to the manufacturer's instructions.

\section{Luciferase reporter assay}

The wild-type sequence and mutants in binding sites of circRNA_101505 was cloned downstream of FL reporter vector. Hep3B cells were seeded and cultured in 96-well plates for $24 \mathrm{~h}$. The cells were co-transfected with FL reporter, Renilla luciferase reporter and miR-103 mimic for $48 \mathrm{~h}$. Luciferase activity was measured using a Luciferase Reporter Assay Kit (BioVision Technologies, Inc, Exton, PA, USA). Relative luciferase activity was normalized to Renilla activity.

\section{RNA immunoprecipitation (RIP)}

A Magna RIP ${ }^{\text {ma }}$ RNA-Binding Protein Immunoprecipitation Kit (Merck Life Science (Shanghai) Co., Ltd. Shanghai, China) was used in this assay as previously describe (22234798). Briefly, Hep3B cells were fixed with formaldehyde, lysed with lysis buffer and sonicated. The supernatant was collected and incubated with a circRNA_101505-specific probes dynabeads (Invitrogen) mixture overnight at $30^{\circ} \mathrm{C}$. On the next day, the dynabeads was washed and incubated with $200 \mu \mathrm{l}$ of lysis buffer. Finally, RNA was extracted from these complexes and was used for qPCR.

\section{Tumor xenograft in nude mice}

Animal experiments were approved by the Ethical Committee for Animal Research of the Third Xiangya Hospital of Central South University. Ten nude mice (5 mice per group, male, 2 months old) were purchased from Animal Center of Central South University. The Hep3B-R cells transfected with circRNA_101505-OE was subcutaneously injected into mice. The tumor sizes were measured every week. The tumor volume was calculated using the formula: $0.5 \times L \times W^{2}$ where $\mathrm{L}$ and $\mathrm{W}$ are the long and short diameter of the tumor, respectively.

\section{Immunohistochemistry}

The expression of Ki67 in tumor tissues from nude mice was analyzed by immunohistochemical analysis. Briefly, the tissues were fixed with $4 \%$ formaldehyde for $24 \mathrm{~h}$, embedded and cut into 4- $\mu \mathrm{m}$-thick section. The sections were treated with $10 \mathrm{mmol} / \mathrm{l}$ sodium citrate buffer and incubated with anti-Ki67 antibody (1:200 dilution) overnight at $4{ }^{\circ} \mathrm{C}$. The positive signaling was stained by using a Mouse and Rabbit Specific HRP/DAB (ABC) Detection IHC kit (Abcam Trading (Shanghai) Company Ltd., Shanghai, China), and counterstained with hematoxylin. The relative integral optical density (IOD) of positive signaling was obtained by ImageJ software.

\section{Statistical analysis}

Data were analyzed in GraphPad Prism software (GraphPad Software Inc., La Jolla, CA). Overall survival analysis was performed by Kaplan-Meier curves and logrank test for significance. Student's $t$ test with two tails was used to assess the statistical significance in two groups and one-way ANOVA with post hoc Bonferroni test were used in three or more groups. Correlations were analyzed by Pearson correlation test. $P<0.05$ was considered statistically significant.

\section{Acknowledgements}

This work was supported by National Natural Science Foundation of China (No. 81573091 and No. 81802668) and Natural Science Foundation of Hunan Provincial China (No. 2018JJ3776 and No. 2017JJ3467).

\section{Authors Contributions}

F.L., X.N. and R.G. conceived the study and participated in the study design, performance, coordination and manuscript writing. Y.L., Y.F., R.H. and M.G. performed the research. All authors have read and approved the final manuscript. In Figure 1, Y.L. generated the data and assembled the figure. In Figure 2, R.H. generated the data and labelled the image, Y.L. and X.N. assembled the figure. In Figure 3, Y.F. and R.H. generated the data and labelled the image, Y.L. and X.N. assembled the figure. In Figure 4, F.L. and R.G. generated the data and labelled the image, Y.L. assembled the figure. In Figure 5, F.Y., F.L. and R.G. generated the data and labelled the image, F.L. and R.G. assembled the figure.

Competing interests

The authors declare no competing interests. 


\section{Publisher's note}

Springer Nature remains neutral with regard to jurisdictional claims in published maps and institutional affiliations.

Received: 23 May 2019 Revised: 24 June 2019 Accepted: 26 June 2019 Published online: 29 July 2019

\section{References}

1. Wang, M., Yu, F. \& Li, P. Circular RNAs: characteristics, function and clinical significance in hepatocellular carcinoma. Cancers 10, pii: E258 (2018).

2. Fu, L., Jiang, Z., Li, T., Hu, Y. \& Guo, J. Circular RNAs in hepatocellular carcinoma: Functions and implications. Cancer Med. 7, 3101-3109 (2018).

3. Qiu, L. P. et al. The emerging role of circular RNAs in hepatocellular carcinoma. J. Cancer 9, 1548-1559 (2018).

4. $\mathrm{Hu}$, J. et al. Progress and prospects of circular RNAs in Hepatocellular carcinoma: novel insights into their function. J. Cell Physiol. 233, 4408-4422 (2018).

5. Yao, R., Zou, H. \& Liao, W. Prospect of circular RNA in hepatocellular carcinoma: a novel potential biomarker and therapeutic target. Front Oncol. 8, 332 (2018).

6. Guo, J., Duan, H., Li, Y., Yang, L. \& Yuan, L. A novel circular RNA circ-ZNF652 promotes hepatocellular carcinoma metastasis through inducing snailmediated epithelial-mesenchymal transition by sponging miR-203/miR-5025p. Biochem. Biophys. Res. Commun. 513, 812-819 (2019).

7. $\mathrm{Xu}$, L. et al. CircSETD3 (Hsa_circ_0000567) acts as a sponge for microRNA-421 inhibiting hepatocellular carcinoma growth. J. Exp. Clin. Cancer Res. 38, 98 (2019).

8. Matboli, M. et al. circRNAs (hsa_circ_00156, hsa_circ_000224, and hsa_circ _000520) are novel potential biomarkers in hepatocellular carcinoma. J. Cell Biochem. 38, BSR20180915 (2018).

9. Ding, B., Lou, W., Xu, L. \& Fan, W. Non-coding RNA in drug resistance of hepatocellular carcinoma. Biosci. Rep. 38, pii: BSR20180915 (2018).

10. Zhu, $\mathrm{H}$. et al. Molecular mechanisms of cisplatin resistance in cervical cancer. Drug Des. Devel. Ther. 10, 1885-1895 (2016).

11. Ghosh, S. Cisplatin: the first metal based anticancer drug. Bioorg. Chem. 88, 102925 (2019).

12. Sun, C. Y., Zhang, Q. Y., Zheng, G. J. \& Feng, B. Phytochemicals: current strategy to sensitize cancer cells to cisplatin. Biomed. Pharm. 110, 518-527 (2019).
13. Su, M. et al. Circular RNAs in cancer: emerging functions in hallmarks, stemness, resistance and roles as potential biomarkers. Mol. Cancer 18, 90 (2019).

14. Kun-Peng, Z., Xiao-Long, M. \& Chun-Lin, Z. Overexpressed circPVT1, a potential new circular RNA biomarker, contributes to doxorubicin and cisplatin resistance of osteosarcoma cells by regulating ABCB1. Int. J. Biol. Sci. 14, 321-330 (2018).

15. Huang, X. et al. Circular RNA AKT3 upregulates PIK3R1 to enhance cisplatin resistance in gastric cancer via miR-198 suppression. Mol. Cancer 18, 71 (2019).

16. Chi, B. J. et al. Downregulation of hsa_circ_0000285 serves as a prognostic biomarker for bladder cancer and is involved in cisplatin resistance. Neoplasma 66, 197-202 (2019).

17. Feng, W. et al. ncRNAs associated with drug resistance and the therapy of digestive system neoplasms. J. Cell Physiol. (2019) https://doi.org/10.1002/ jcp.28551.

18. Wan, L. et al. MicroRNA-103 confers the resistance to long-treatment of adriamycin to human leukemia cells by regulation of COP1. J. Cell Biochem. 119, 3843-3852 (2018).

19. Garofalo, M. et al. EGFR and MET receptor tyrosine kinase-altered microRNA expression induces tumorigenesis and gefitinib resistance in lung cancers. Nat. Med. 18, 74-82 (2011).

20. Zhang, Z. et al. circARF3 alleviates mitophagy-mediated inflammation by targeting miR-103/TRAF3 in mouse adipose tissue. Mol. Ther. Nucleic Acids 14 192-203 (2019).

21. Nie, $X$. et al. Cloning, expression, and mutation analysis of NOR1, a novel human gene down-regulated in HNE1 nasopharyngeal carcinoma cell line. J. Cancer Res. Clin. Oncol. 129, 410-414 (2003).

22. Wang, W. et al. Oxidored-nitro domain containing protein 1 (NOR1) expression suppresses slug/vimentin but not snail in nasopharyngeal carcinoma: Inhibition of EMT in vitro and in vivo in mice. Cancer Lett. 348, 109-118 (2014).

23. Shan, Z. et al. Overexpression of oxidored-nitro domain containing protein 1 induces growth inhibition and apoptosis in human prostate cancer PC3 cells. Oncol. Rep. 32, 1939-1946 (2014).

24. Gui, R., Li, D., Qi, G., Suhad, A. \& Nie, X. Inhibition of Grb2-mediated activation of MAPK signal transduction suppresses NOR1/CB1954-induced cytotoxicity in the HepG2 cell line. Oncol. Lett. 4, 566-570 (2012). 